\title{
Twins born in different environments? Nuclei of two dSphs: isolated galaxy KKS3 and E269-66, a close neighbor of NGC5128
}

\author{
Margarita Sharina ${ }^{1}$, Alexei Kniazev ${ }^{2,3,4}$ and Igor Karachentsev ${ }^{1}$ \\ ${ }^{1}$ Special Astrophysical Observatory, Russian Academy of Sciences, Nizhnii Arkhyz, 369167, \\ Russia \\ ${ }^{2}$ South African Astronomical Observatory, PO Box 9, Observatory 7935, Cape Town, \\ South Africa \\ ${ }^{3}$ Southern African Large Telescope Foundation, PO Box 9, Observatory 7935, Cape Town, \\ South Africa \\ ${ }^{4}$ Sternberg Astronomical Institute of Lomonosov Moscow 119992, Russia \\ email: sme@sao.ru, akniazev@saao.ac.za, ikar@sao.ru
}

\begin{abstract}
We present the results of age, metallicity and radial velocity determination for central massive globular clusters (GCs) in dwarf spheroidal galaxies: KKs3 and ESO269-66. KKS3 is a unique isolated galaxy. ESO269-66 is a close neighbor of the giant S0 Centaurus A. The results contribute to the knowledge about the origin of massive star clusters and their host dSphs. The structure and star formation histories of the two dwarf galaxies look rather similar. Both of them have experienced several star-forming events. The most recent ones occurred 1-2 Gyr ago, and most powerful bursts happened 12-14 Gyrs ago. Our analysis has shown that both GCs appear to be 1-2 Gyr younger and 0.1-0.3 dex more metal-rich than the most ancient metal-poor stars in the host dSphs. We examine signatures of multiple stellar population in the GCs using our data. Since central star-forming bursts were extended in time, the massive clusters might be considered as nuclei of the galaxies.
\end{abstract}

Keywords. galaxies: dwarf: individual (KKs3, ESO269-66) - galaxies: nuclei, formation

The close vicinity of giant neighbors determines the environmental mechanisms that have been considered responsible for the evolution of dwarf spheroidal galaxies (dSphs). In the recent years, Karachentsev and collaborators have reported on the discovery of a few truly isolated dSphs in the Local volume. This study focuses on one of these unusual objects, KKs3. It contains a massive globular cluster near its optical center. We have estimated its radial velocity using a medium-resolution spectrum obtained with the RSS spectrograph at the Southern African Large Telescope (SALT). The signal-to-noise ratio in the spectrum was sufficient to estimate the age and metallicity for the GC using simple stellar population models and the method of Lick index diagnostic diagrams (Worthey et al. /, 1994; Worthey \& Ottaviani, 1997). In the same way we have analyzed another

Table 1. Main parameters of the studied galaxies and their globular clusters.

\begin{tabular}{|lccccc|llllll|}
\hline $\begin{array}{l}\text { Dwarf } \\
\text { galaxy }\end{array}$ & $\begin{array}{c}\text { Distance } \\
{[\mathrm{Mpc}]}\end{array}$ & $\begin{array}{c}\text { Diameter } \\
{[\mathrm{kpc}]}\end{array}$ & $\begin{array}{c}M_{V} \\
{[\mathrm{mag}]}\end{array}$ & $\begin{array}{c}\mathrm{V}-\mathrm{I} \\
{[\mathrm{mag}]}\end{array}$ & $\begin{array}{c}{[\mathrm{Fe} / \mathrm{H}]^{12-14} \text { Gyr }} \\
{[\mathrm{dex}]}\end{array}$ & $\begin{array}{l}\text { G C } \\
\text { in }\end{array}$ & $\begin{array}{c}M_{V} \\
{[\mathrm{mag}]}\end{array}$ & $\begin{array}{c}\mathrm{V}-\mathrm{I} \\
{[\mathrm{mag}]}\end{array}$ & $\begin{array}{c}{[\mathrm{Fe} / \mathrm{H}]} \\
{[\mathrm{dex}]}\end{array}$ & $\begin{array}{c}\mathrm{Age} \\
{[\mathrm{Gyr}]}\end{array}$ & $\begin{array}{c}V_{h} \\
{[\mathrm{~km} / \mathrm{s}]}\end{array}$ \\
\hline KKs3 & $2.12^{4}$ & $1.5^{3}$ & $-12.5^{3}$ & $0.85^{3}$ & $-1.90^{4}$ & KKs3 & $-8.5^{4}$ & $0.83^{4}$ & $-1.55^{1}$ & $12^{1}$ & $316 \pm 7^{3}$ \\
E269-66 & $3.82^{5}$ & $1.5^{5}$ & $-14.4^{5}$ & $0.94^{5}$ & $-1.75^{6}$ & E269-66 & $-9.9^{2}$ & $0.93^{2}$ & $-1.65^{1}$ & $12^{1}$ & $775 \pm 6^{1}$
\end{tabular}

Notes: 1: This work; 2: Georgiev et al. (2009); 3: Karachentsev et al. (2015a); 4:Karachentsev et al. (2015b)); 5: Karachentsev et al. (2007); 6: Makarova et al. (2007). 

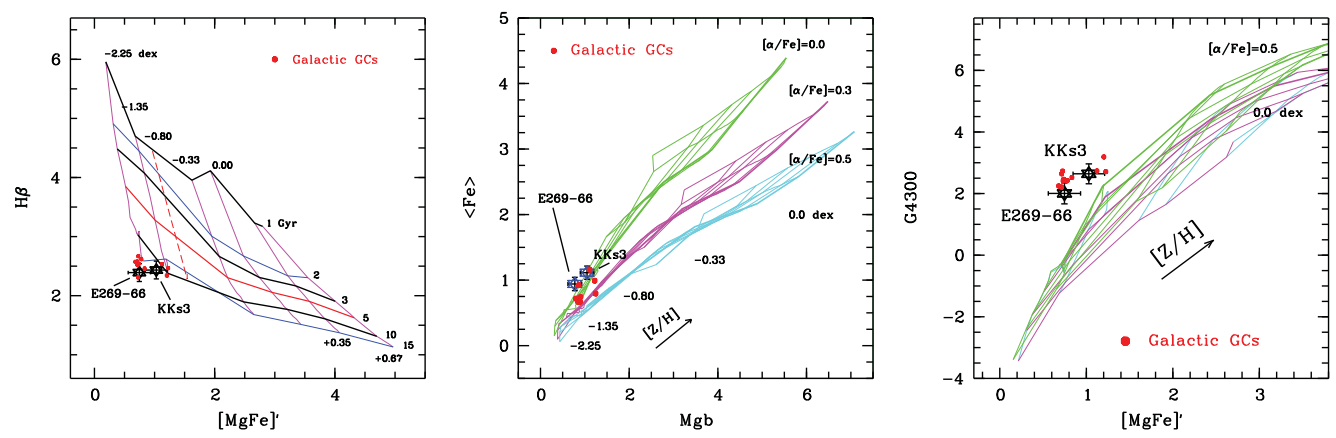

Figure 1. Lick-index diagnostic plots for GCs in KKs3 and E269-66: age vs. metallicity and $[\alpha / \mathrm{Fe}]$ vs. metallicity. SSP models by Thomas et a. $(2003,2004)$ are overplotted.

luminous GC in the center of ESO269-66. The cluster was observed with SALT in the same instrumental configuration.

Medium-resolution spectroscopic analysis is a promising tool for breaking the agemetallicity degeneracy and studying light element abundance anomalies using integratedlight spectra of globular clusters (e.g. Schiavon et al. /, 2012, see also Sharina et al. (2010) for the description of the used methods). We selected Galactic GCs with the values of the Lick indices (Schiavon et al. /, 2012) close to the ones of our two GCs: NGC1904, NGC3201, NGC5946, NGC5986, NGC6254, NGC6752, NGC7089. Fig. 1 illustrates the similarity of the age, metallicity and light-element sensitive indices of Galactic GCs and the two nuclei of dSphs. Many of the selected Galactic GCs have blue horizontal branches, host multiple stellar populations and show light element abundance variations. The resulting ages, metallicities and radial velocities for GCs in KKs3 and E269-66, and photometric and spectroscopic data from the literature are summarized in Table 1. Star formation histories of KKs3 and E269-66 look similar. Determination of the detailed spectroscopic abundances of their nuclei is a subject of future studies.

\section{Acknowledgements}

This work is supported by the Russian Scientific Foundation grant 14-12-00965. A.Y.K. acknowledges the support from the National Research Foundation (NRF) of South Africa. Based on SALT observations under the program 2015-1-SCI-030.

\section{References}

Georgiev, I. Y., Hilker, M., Puzia, T. H., Goudfrooij, P., \& Baumgardt, H. 2009, MNRAS, 396, 1075

Karachentsev, I. D., Kniazev, A. Yu., \& Sharina, M. E. 2015a, Astron.Nachr, 336, 707

Karachentsev, I. D., Makarova, L. N., Makarov, D. I., Tully, R. B., \& Rizzi, L. 2015b, MNRAS (Letters), 447, 85

Karachentsev, I. D., et al. 2007, AJ, 133, 504

Makarova, L. N., et al. 2007, in: F. Combes and J. Palous (eds.) Galaxy Evolution Across the Hubble Time, Proc. IAUS 235 (Cambridge: Cambridge University Press), p. 320

Schiavon, R. P., Caldwell, N., Morrison, H., Harding, P., Courteau, S., MacArthur, L. A., \& Graves, G. J. 2012, AJ, 143, 14

Sharina, M. E., Chandar, R., Puzia, T. H., Goudfrooij, P., \& Davoust, E. 2010 MNRAS, 405, 839

Thomas, D., Maraston, C., \& Korn, A. 2004, MNRAS (Letters), 351, 19

Thomas, D., Maraston, C., \& Bender, R. 2003, MNRAS, 343, 279

Worthey, G., \& Ottaviani, D. L. 1997, ApJS, 111, 377

Worthey, G., Faber, S. M., Gonzalez, J. J., \& Burstein, D. 1994, ApJS, 94, 687 